\title{
Simulation Based Performance Evaluation of Queueing Disciplines for Multi-Class Traffic
}

\author{
${ }^{1}$ Saptarshi Ray, ${ }^{2}$ Satyajit Panigrahi \\ ${ }^{1,2}$ (B.Tech, Computer Science and Engineering, Veer Surendra Sai University of Technology, Burla, India)
}

\begin{abstract}
Queuing is one of the very vital mechanisms in traffic management system. Each router in the network must implement some queuing discipline that governs how packets are buffered while waiting to be transmitted. Several queue scheduling disciplines have been designed to improve the quality of service and to avoid congestion for networked multimedia applications. In this paper, we evaluate the performance of four queueing disciplines (FIFO, PQ,WFQ and DWRR) that are widely deployed for multi-class traffic and analyze their performance using OPNET simulator and compare their relative performance based on queueing delay, packet received, packet drop rate, end-to-end delay and delay jitter with RED and WRED policies.
\end{abstract}

Keywords - Deficit-Weighted Round Robin, Multi-Class Traffic, OPNET Simulation, Priority Queuing, Weighted-Fair Queuing.

\section{Introduction}

With the growth of internet during the last decade IP networks are expected to support not only typical services like ftp and email but also video streaming and voice applications. There are many network parameters for its performance evaluation that are required for different application A number of queueing disciplines have been proposed to achieve the required quality of service in today's network services. In this paper, we analyze the performance of a typical network topology under four different queueing disciplines which are widely deployed and compare their performance OPNET simulator [2]. The queueing disciplines considered are: FirstIn-First-Out (FIFO), Priority Queueing (PQ), Weighted Fair queueing (WFQ) and Deficit Weighted Round Robin queueing (DWRR). We also scrutinize the effect of two dropping policies, random-early drop (RED) [3] and weighted random-early drop(WRED), on their performance. We then present the simulation results and evaluate their performance.

\section{Queue Scheduling Disciplines}

We have selected four popular queuing disciplines for our experiments: FIFO, PQ, WFQ and DWRR. We describe them one by one here.

First-In-First-Out (FIFO) queueing is the most basic queueing discipline. This expression describes the principle of a queue or firstcome-firstserve behavior. In FIFO queueing, all packets placed into a single queue and then served in the same order on which they arrived. The behavior of a FIFO queue is very predictablepackets are not reordered and the maximum delay is determined by the maximum depth of the queue. For software based routers. A bursty flow can consume the entire buffer space of a FIFO queue, and that causes all other flows to be denied service until after the burst is serviced word processor, please use the font closest in appearance to Times. Avoid using bit-mapped fonts if possible. True-Type 1 or Open Type fonts are preferred. Please embed symbol fonts, as well, for math, etc.

Priority Queuing (PQ) assigns multiple queues to a network interface with each queue being given a priority level. Packets of different flows are assigned a priority level according to their Quality of Service (QoS) requirements. In PQ, packets that arrive at the output link are classified into more than one queues based on their priorities. The packets with highest priority are then served first, next second higher priority and so on, queues are served in order. Packets in the same priority class are serviced in a FIFO manner. The main benefit of PQ is that packets of different classes can be managed using different queues and, consequently, a certain class of traffic can be handled in different way than another one. The limitation of PQ is that lower-priority packets may receive little attention when a higher-priority class has a continuous stream of packets. This problem is known as starvation problem [4].

Weighted Fair Queueing (WFQ) [5] is an attractive technique decent queuing technique. The main goal behind its proposal was to ensure fairness among all types of traffic. Similar to PQ, arriving packets are first classified and assigned to one of per-class queues based on information taken from the packet header. Each queue is assigned a weight based on the required bandwidth. It is computationally complex and the supported delay bounds can still be large [6]. . The main benefit of WFQ is that it provides a minimum level of network resources to each configured service class. WFQ presents scalability problems and cannot be used in 
Simulation Based Performance Evaluation of Queueing Disciplines for Multi-Class Traffic

environments with high volume of traffic requiring many classes of service. One possible application of WFQ is where the number of service classes is limited to a small number, so that the computational load is bounded.

Deficit Weighted Round Robin (DWRR), is a modified weighted round robin scheduling discipline. Deficit Weighted Round Robin (DWRR) was proposed to guarantee QoS with respect to delay and bandwidth for real-time burst traffic [7]. Similar to WFQ, packets are first classified into various service classes and assigned to separate queues then serviced in a round-robin order. However, each queue maintains a deficit counter that specifies the total number of bytes that the queue is permitted to transmit. Each time a queue is visited by the scheduler the deficit counter is first increased by a quantum of service proportional to its weight. Then, the scheduler transmits the packets at the head of the queue and decrement the deficit counter by the size of the transmitted packet as long as the size of the packet is not greater than the deficit counter. If the queue becomes empty, the deficit counter is set to zero. Then, the scheduler moves to the next non-empty queue. DWRR [6,7] has several strengths over other queue disciplines such as lower complexity and better control of network resources especially when serving variable-size packets.

\section{Packet Dropping Policies}

Random Early Drop (RED) is an active queue management algorithm. It is also a congestion avoidance algorithm. RED monitors the average queue size and drops packets based on statistical probabilities. If the buffer is almost empty, all incoming packets are accepted. As the queue grows, the probability for dropping an incoming packet grows too. When the buffer is full, the probability has reached 1 and all incoming packets are dropped.

Weighted random early detection (WRED) is a queue management algorithm with congestion avoidance capabilities. WRED combines the capabilities of the RED algorithm with the IP Precedence feature to provide for preferential traffic handling of higher priority packets. WRED can selectively discard lower priority traffic when the interface begins to get congested. By dropping some packets early rather than waiting until the queue is full, WRED avoids dropping large numbers of packets at once. Thus, WRED allows the transmission line to be used fully at all times.

\section{Simulation Model}

In order to demonstrate the performance of different queueing disciplines, we considered a hypothetical network topology as illustrated in Figure 1 in our simulations. There are two routers connected by a DS1 link and all other links are 10BaseT. There are four different traffic scenarios, one for each queue scheme. There are three types of traffic :FTP, Voice and Video. There is a separate server for each traffic type. To evaluate and compare the performance of different queueing disciplines, we collected the average queueing delay, the average packet received, the average end-to-end delay, the average packet drop-rate and the average delay jitter for each queue scheme. We also studied the effect of using weighted random early drop(WRED) and tested its dominance over random early drop(RED).

For FTP traffic, we used exponential distribution for packet arrival, constant packet size and best-effort type of service. For video traffic, we used low resolution video starting at $10 \mathrm{fps}$ (frames per sec) arrival rate and $128 \times 120$ pixels and keep increasing this rate and size as load increases. The type of service (ToS) is Streaming Multimedia. For voice traffic, the voice encoder scheme is G.711, the silence and talk spurt lengths are exponentially distributed and ToS is Interactive Voice. All these settings were made using OPNET Application Attributes Profile.

For queueing disciplines specification we set the maximum queue size to be 500 packets. We imply OPNET default weights in case of WFQ and DWRR. There are four types of classes in all queues (except FIFO) whose queue buffer size increases from highest priority to lowest one.

When RED is enabled, we used minimum and maximum threshold as 100 and 200 respectively while keeping the mark probability denominator (the fraction of packets dropped when the average queue size is at maximum threshold) as 10 . We set exponential weight factor (used to calculate average queue size based on the previous average and current queue size) to be 9 .

When WRED is enabled, we used minimum and maximum threshold as 100 and 200 respectively while keeping the mark probability denominator (the fraction of packets dropped when the average queue size is at maximum threshold) as 10 . We set exponential weight factor (used to calculate average queue size based on the previous average and current queue size) to be 9 . 


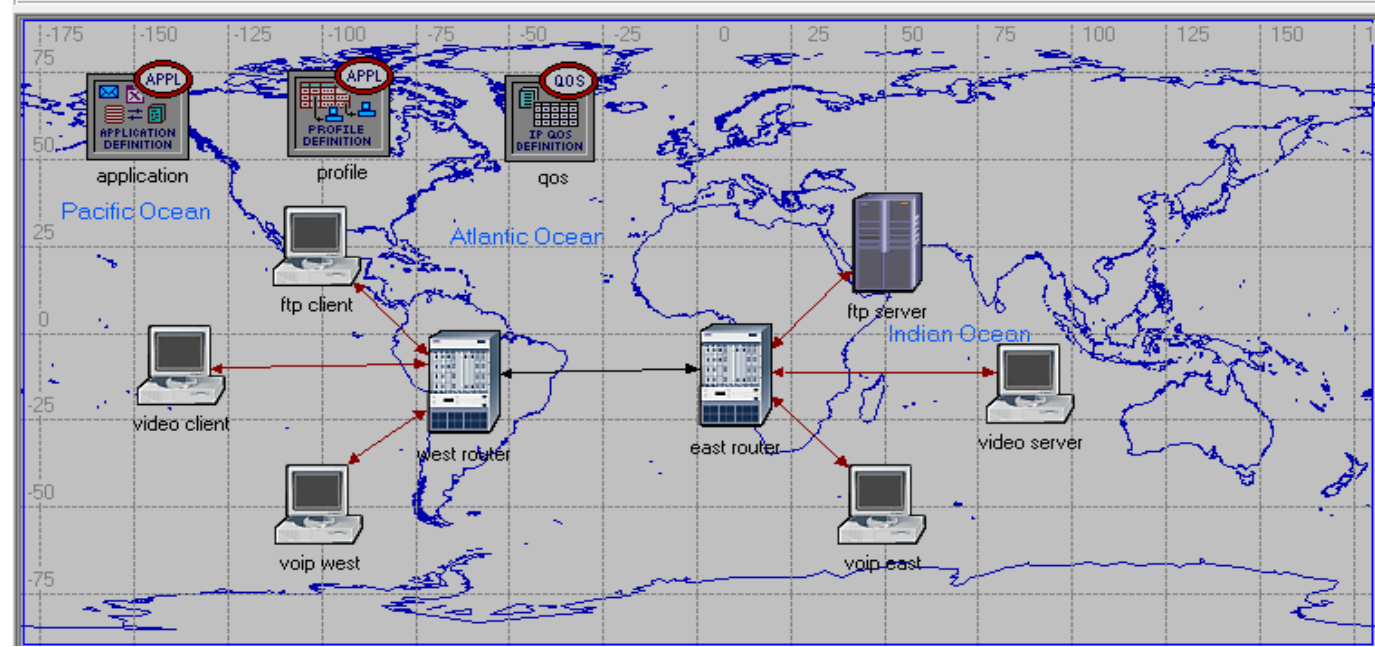

Figure 1. Network topology for simulation

\section{Simulation Results And Performance Comparison}

\subsection{Queueing delay}

Figures (Fig.:2.a,2.b,) shows the average queueing delay of the four queue disciplines RED policy versus simulation time. We can see that FIFO has the worst behavior as compared to other disciplines. As shown in respective figures, WRED has greatly improved the performance of FIFO as compared to the previous results with RED policy. There is no significant improvement for other queueing disciplines in terms of queueing delay. We can see that DWRR gives better performance than other queue disciplines.
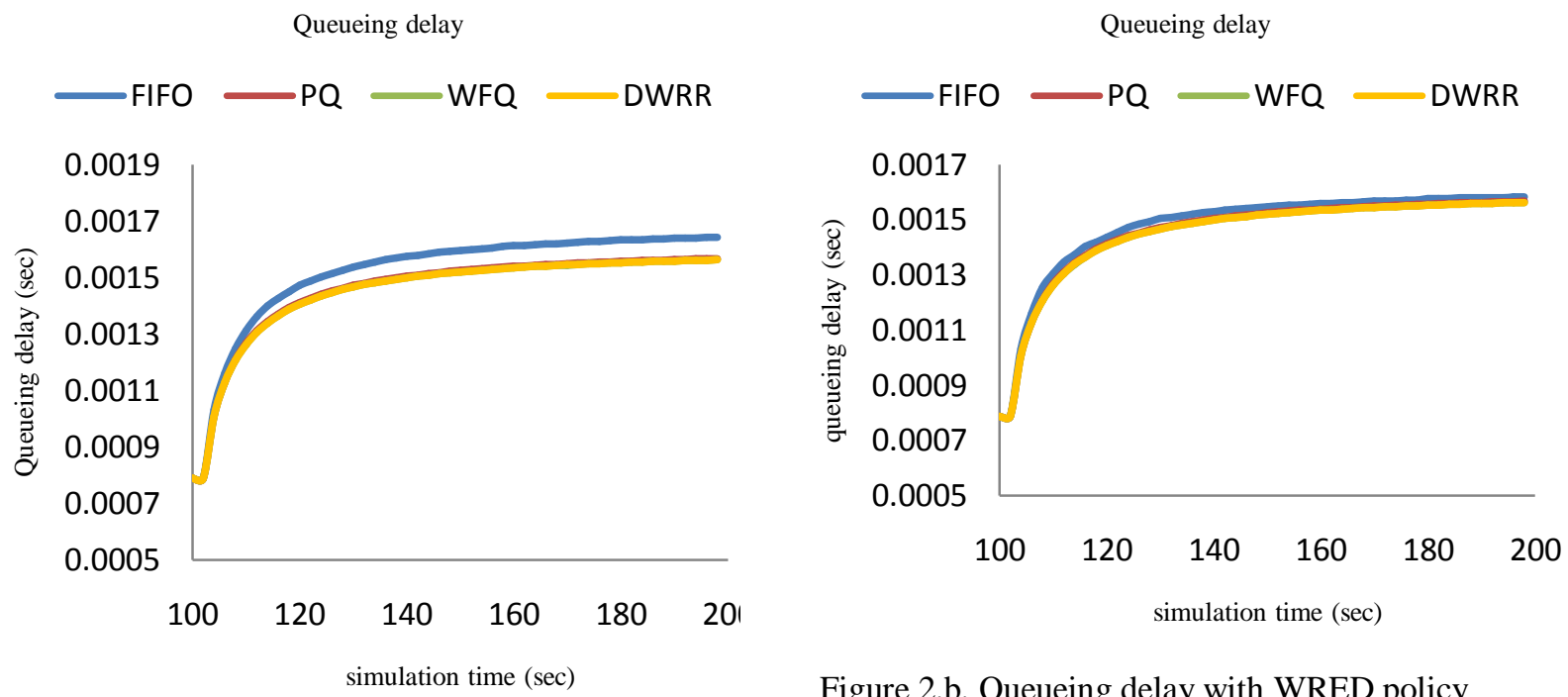

Figure 2.b. Queueing delay with WRED policy

Figure 2.a. Queueing delay with RED policy

\subsection{IP Traffic dropped}

Figures (Fig.: 3.a, 3.b) shows IP traffic dropping statistics, where it can be observed that in the cases of FIFO and PQ the packet drop starts at near 105 sec. Using RED policy Packet drop for FIFO is much higher than others queue disciplines, packet drop for PQ is semi lower and the packet drop for WFQ and DWRR are lower. Using WRED policy Packet drop for FIFO is lowered from more than 40 packets/sec (using RED policy) to more than 18 packets/sec (using WRED policy). However there is no significant improvement for other queueing disciplines in terms of IP traffic drop rate. From figures we can see that DWRR gives better performance than other queue disciplines. 


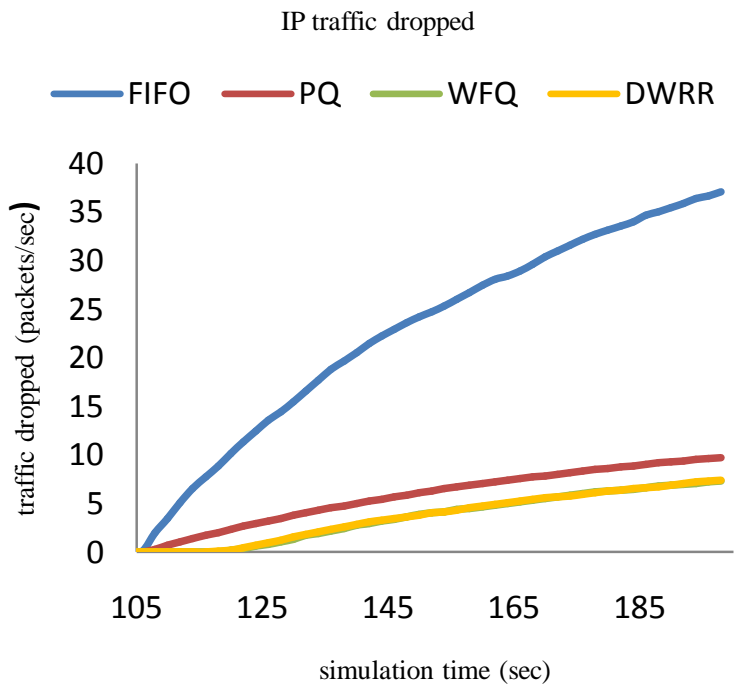

Figure 3.a. IP traffic dropped with RED policy

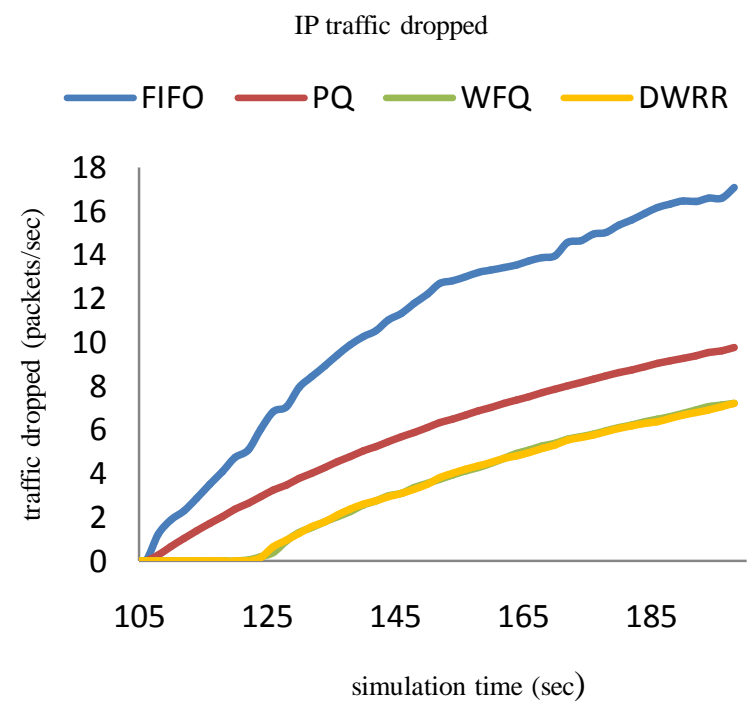

Figure 3.b. IP traffic dropped with WRED policy

\subsection{FTP Traffic Received}

Figures (Fig: 4.a,4.b) shows traffic received statistics for FTP,where it can be observed that as the time increased the file receiving performance graph line for WFQ and DWRR is same in both RED policy and WRED policy .PQ shows zero traffic received for the whole simulation time using both the policies. Using WRED policy the FTP traffic receiving rate of FIFO is down to zero relative to RED policy .Here WFQ and DWRR shows better performance among other queues.

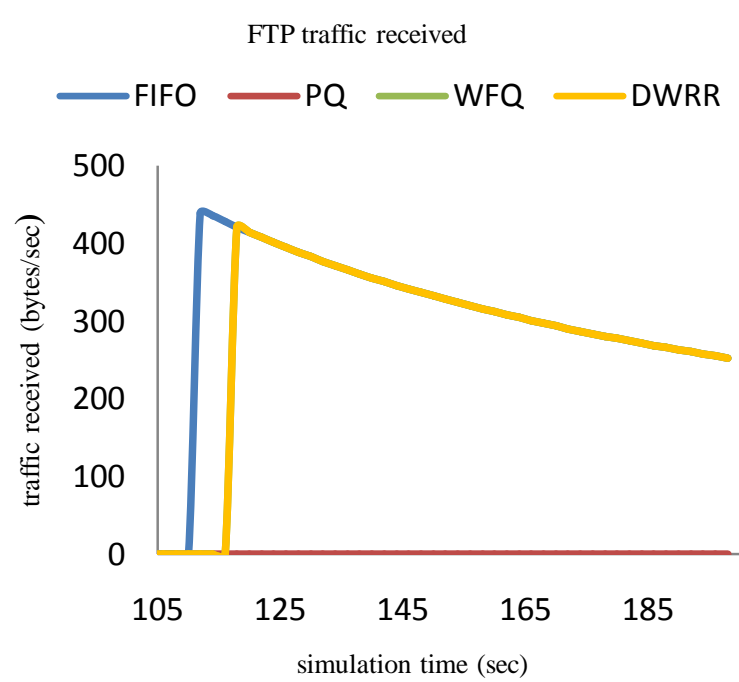

Figure 4.a. FTP traffic received with RED policy

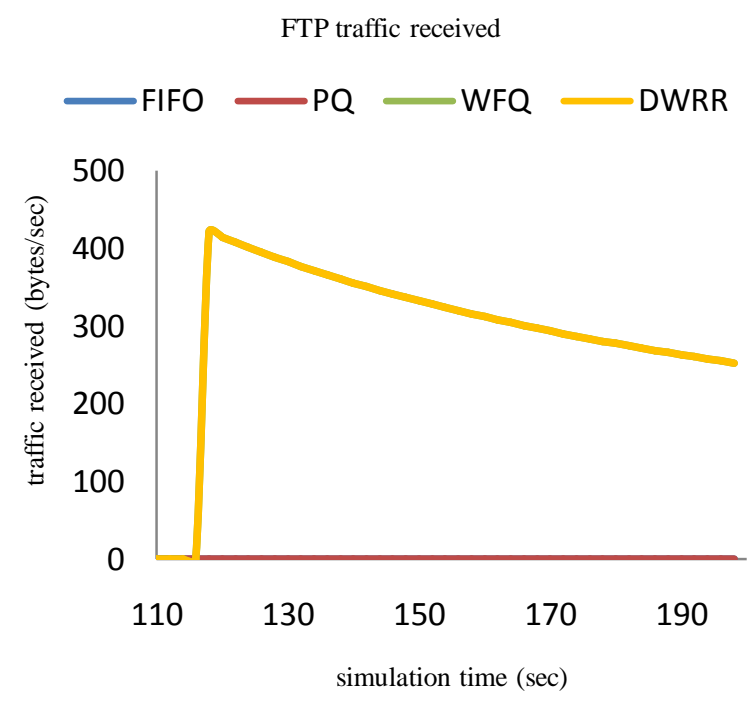

Figure 4.b. FTP traffic received with WRED policy

\subsection{Video Conferencing Traffic Received}

Figures (Fig.: 5.a, 5.b) shows traffic Received statistics for Video conferencing, where it can be observed that in cases both of both the policies video receiving rate graph of WFQ and DWRR group is always higher than the performance graph of FIFO group and PQ group is lower than the other groups. Using WRED policy there is increase in traffic receiving rate of FIFO relative to RED policy. The traffic receiving rate of WFQ has increased than DWRR relative to using RED policy. 
Video conferencing traffic received

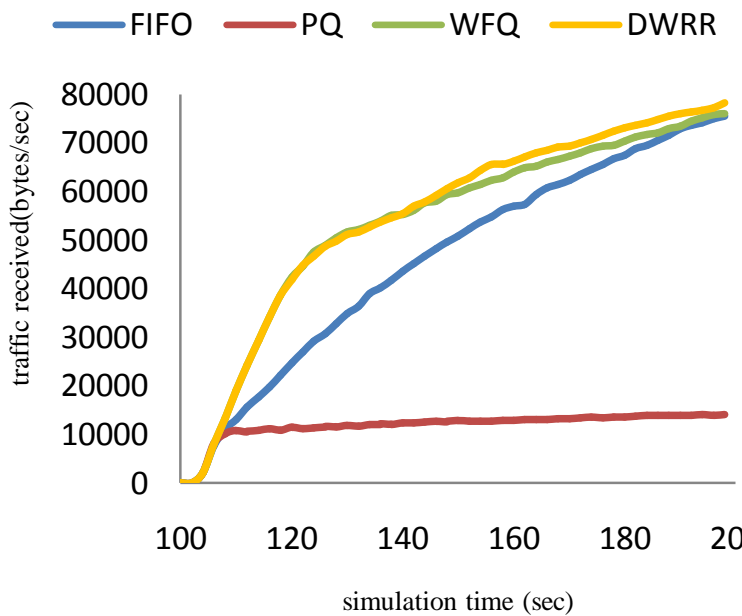

Figure 5.a. Video conferencing traffic received with RED
Video conferencing traffic received

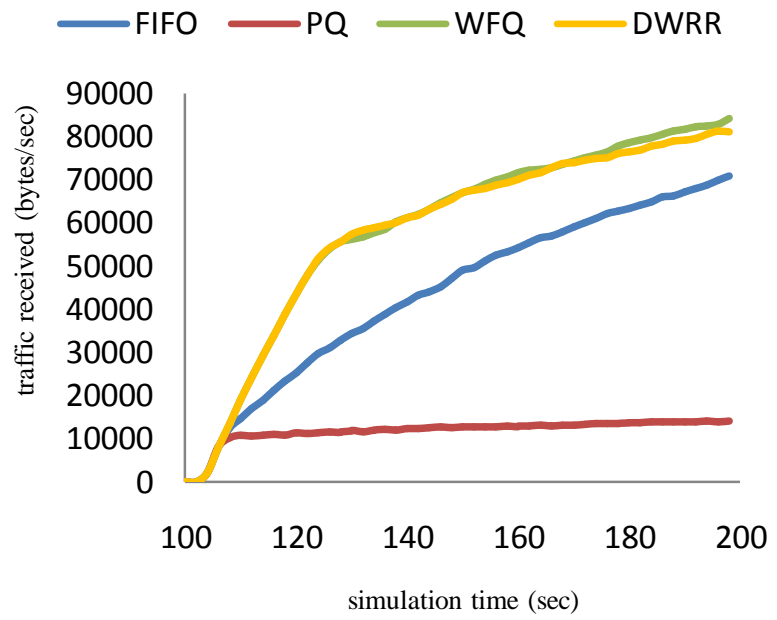

Figure 5.b. Video conferencing traffic received with WRED

\subsection{Video Conferencing Packet end to end Delay}

Figures (fig: 6.a, 6.b) shows Packet end to end delay time vs. simulation time for Video conferencing, where it can be observed that there is improvement in FIFO and WFQ showed in WRED output for end-to-end delay relative to RED policy. We also noticed that WFQ and DWRR do not produce good results for this type of performance measures when compared with even FIFO. PQ outperforms all other queueing disciplines in terms of end-to-end delay.
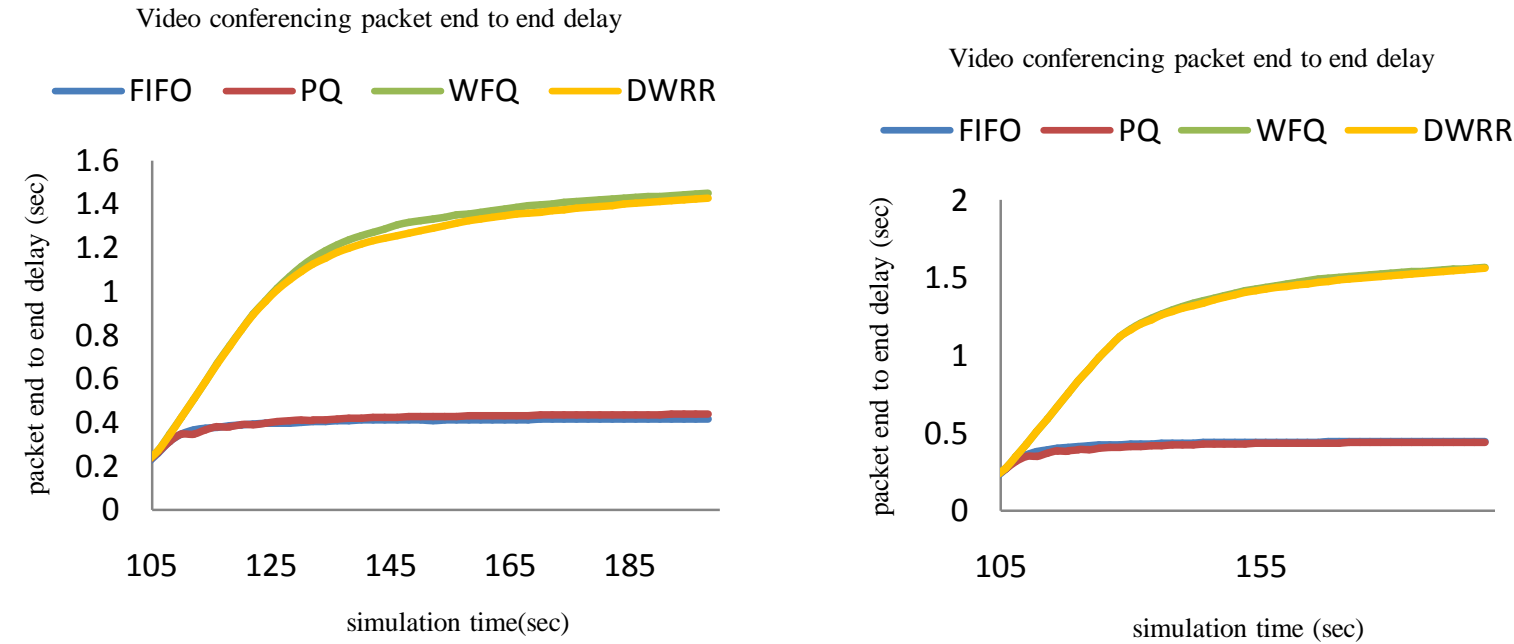

With RED policy end delay with WRED policy

Figure 6.a. Video conferencing packet end to end delay end with RED policy

Figure 6.b. Video conferencing packet end to delay with WRED policy

\subsection{Video Conferencing Delay Jitter}

Figures (fig: 7.a, 7.b)shows Packet delay variation vs. simulation time for Video conference, where it can be observed that DWRR group's Packet delay variation time increases in Video conference. PQ outperforms all other queueing disciplines in terms of delay jitter for video traffic with RED and WRED policies. There is very little improvement in FIFO scheduling for WRED policy relative to RED policy. However there is no improvement for other queue discipline in using WRED policy. 


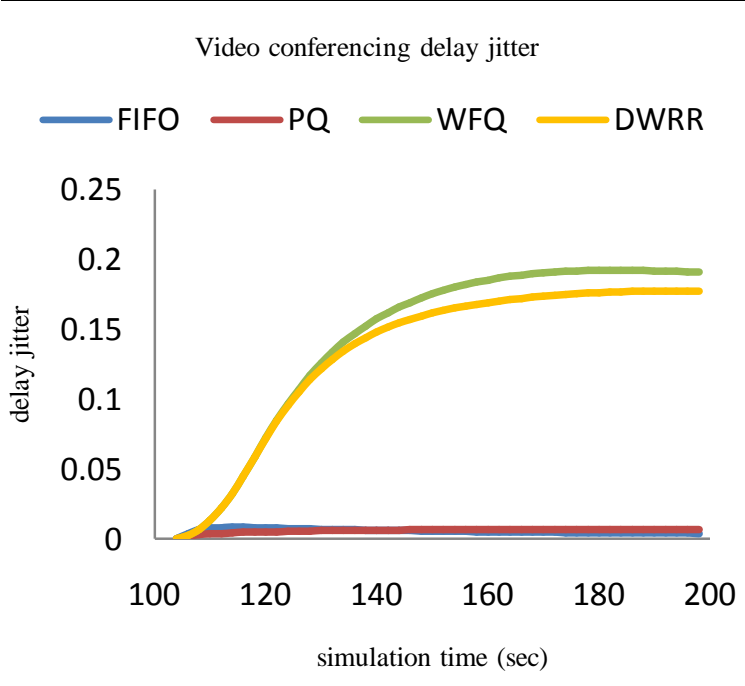

Figure 7.a.Video conferencing delay jitter with RED policy

\subsection{Voice Traffic Received}

Figures (Fig.: 8.a, 8.b) shows traffic Received vs. simulation time for VoIP, where it can be observed that. The performance graph line of FIFO group is always lowered compared to the other groups PQ, WFQ and DWRR which are always same in case of RED and WRED policies. The voice traffic receiving rate in WRED for FIFO is almost same with PQ,WFQ and DWRR relative to RED policy.

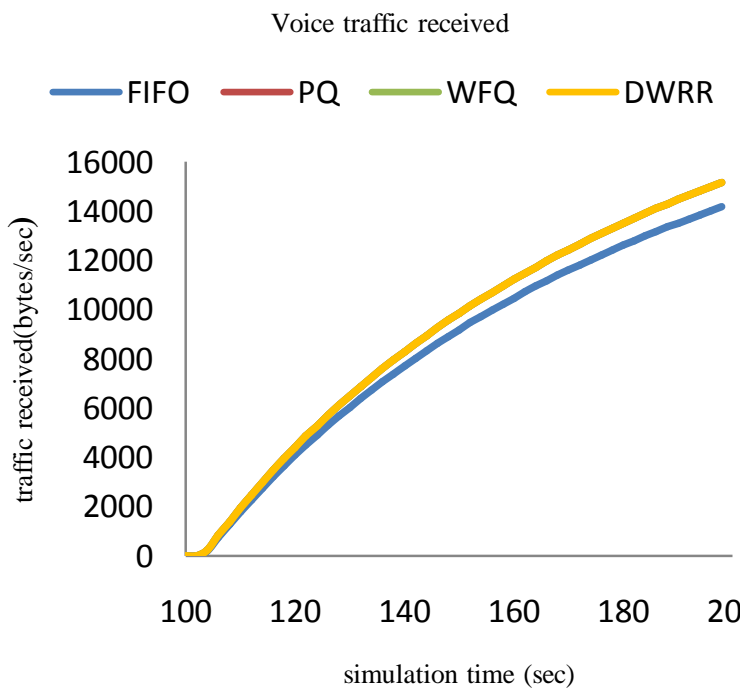

Figure 8.a. Voice traffic received with RED policy

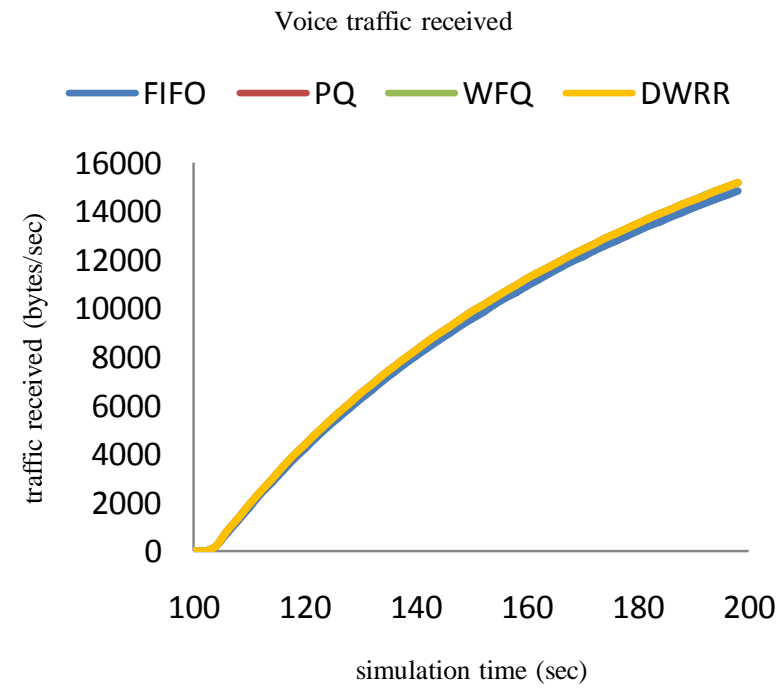

Figure 8.b. Voice traffic received with WRED policy

\section{CONCLUSION}

In this study we presented a simulation-based performance evaluation and comparison of four queueing scheduling disciplines for different traffic sources. We also examined the impact of using weighted random early drop (WRED) as compared to random early drop policy (RED). After the comparison of the results it is clear that the queueing delay of FIFO has been improved relative to RED policy. FIFO group's performance is also better in some cases compared with PQ group. The IP traffic dropped for FIFO is also reduced in using WRED than RED policy. Now, if we compare Queues in case of File receiving, voice data receive and video conferencing, WFQ and DWRR always shows the better performance among them. The simulation results show that DWRR gives the minimum average queuing delay and minimal average packet drop rate for multi-class traffic. Although PQ and WFQ are also very close to it. DWRR and WFQ queuing schemes maintain fairness 
well. PQ outperforms other disciplines in terms of delay jitter and packet end to end delay for video traffic. We also noticed that using WRED has greatly improved all the performance measures especially with FIFO queue discipline in terms of queueing delay. Using WRED policy there is increase in traffic receiving rate for voice traffic of FIFO relative to RED policy. The traffic receiving rate of WFQ for video conferencing has increased than DWRR relative to using RED policy. Further work is still required to carefully examine these queueing disciplines. OPNET provides a very impressive and real environment like picture what happens to the network performance when we change the queuing discipline.

\section{REFERENCES}

[1] Hafiz M. Asif and El-Sayed M. El-Alfy, Simulation-Based Performance Comparison of Queueing Disciplines for Differentiated Services Using OPNET,2005.

[2] OPNET Technologies, Inc.

[3] S. Floyd and V. Jacobson, Random Early Detection Gateways for Congestion Avoidance, IEEE/ACM Trans. on Networking, vol. 1, no. 4, pp. 397-413, 1993.

[4] J. Kurose and K. Ross, Computer Networking: A Top-Down Approach Featuring the Internet, 3/e. Addison Wesley, 2005.

[5] A. Demers, S. Keshav, and S. Shenkar, Analysis and Simulation of a Fair Queueing Algorithm, Proceedings of the ACM SIGCOMM, Austin, TX, pp. 1-12, 1989

[6] C. Semeria, Supporting Differentiated Service Classes: Queue Scheduling Disciplines, White Paper, Juniper Networks, Inc., 2001.

[7] T. Kwon, S. Lee and J. Rho, Scheduling Algorithm for Real-Time Burst Traffic Using Dynamic Weighted Round Robin, Proceedings of the IEEE Int. Symposium on Circuits and Systems, vol. 6, no. 31, pp. 506 509, 1998. 